\title{
Stomach secretes estrogen in response to blood triglyceride levels in males
}

Yoshimitsu Kanai ( $\nabla$ ykanai@wakayama-med.ac.jp)

Wakayama Medical University https://orcid.org/0000-0002-0375-6853

Takao Ito

Wakayama Medical University

Yuta Yamamoto

Wakayama Medical University

Naoko Yamagishi

Wakayama Medical University

\section{Article}

Keywords: Estrogen, lipid homeostasis, blood triglyceride, stomach

Posted Date: March 25th, 2021

DOI: https://doi.org/10.21203/rs.3.rs-289734/v1

License: (c) (1) This work is licensed under a Creative Commons Attribution 4.0 International License.

Read Full License

Version of Record: A version of this preprint was published at Communications Biology on December 7th, 2021. See the published version at https://doi.org/10.1038/s42003-021-02901-9. 


\section{Abstract}

The central nervous system receives body energy information and controls feeding behavior and lipogenesis1. Ghrelin, insulin, leptin and vagal afferents transmit the status of fasting, blood glucose, body fat, and food intake, respectively2-5. Estrogen, which is secreted from adipocytes and gastric parietal cells and from the ovaries in females, also acts upon the central nervous system and liver to inhibit feeding behavior and lipogenesis6-9. How blood triglyceride levels are monitored and how estrogen levels are regulated from the perspective of the lipid homeostasis is not well understood. Using male rats, we show that gastric parietal cells secrete estrogen in response to blood triglyceride levels. Parietal cells predominantly use fatty acid as an energy source. When male rats are administered olive oil or glucose, blood estrogen levels increase as the blood triglyceride, but not glucose, levels rise. Estrogen levels in stomach tissues increase as the blood triglyceride levels rise, and blood triglyceride leveldependent increases of blood estrogen levels are cancelled in gastrectomized rats. We therefore propose that in males, parietal cells in the stomach act as a sensor for the blood triglyceride levels and can secrete estrogen to inhibit the hepatic lipogenesis and feeding behavior when blood triglyceride levels are high.

\section{Main Text}

Estrogen is a multi-target and multi-functional hormone beyond just a sex hormone; it controls glucose/lipid homeostasis, bone metabolism, brain function and skeletal growth as well as follicular growth and ovulation ${ }^{7}$. Aside from the ovaries in females, estrogen is also secreted from adipose tissues, which are greatly increased in males ${ }^{10,11}$. Our previous studies showed that parietal cells in rat stomachs also express aromatase, together with 17a-hydroxylase and 17b-hydroxysteroid dehydrogenase type III (17b-HSD type III), and convert testosterone (male) or progesterone (female) to estrogen (17b-estradiol [E2] $)^{6,12}$ (Fig. 1a). Aromatase was prominently expressed in the stomach among the tissues upstream of the portal vein (stomach, duodenum, jejunum, ileum, colon and pancreas) ${ }^{6}$, and the small amounts of aromatase in the rest of the tissues were mainly from the attached adipose tissues (Fig. 1b, c, Extended Data Fig. 1). Parietal cells secrete estrogen (gastric estrogen) into the portal vein, so estrogen levels are higher in the portal vein (ca. $x 2$ ) than in the artery in male ${ }^{6}$ and female ${ }^{13}$ rats, which is lost in gastrectomized (GX) rats ${ }^{6}$. The role of gastric estrogen and how its secretion is regulated are not widely understood. Here, using male rats, we show that parietal cells act as a sensor for the blood triglyceride levels and gastric estrogen is secreted in response to the blood triglyceride levels. Estrogen suppresses the lipid supply by inhibiting the feeding behavior and the de novo lipogenesis in the liver ${ }^{8,9}$, so we propose that when blood triglyceride levels rise, parietal cells increase the secretion of estrogen, which lowers the blood triglyceride levels to maintain proper blood triglyceride levels in males (Fig. 3d).

Gastric estrogen directly enters the liver via the portal vein, where estrogen controls glucose/lipid homeostasis ${ }^{9}$. This secretion is similar to that of insulin, which is secreted from pancreatic b-cells, enters the portal vein, and directly acts on the liver to keep appropriate blood glucose levels ${ }^{14,15}$. b-cells, which sense the blood glucose levels, use glucose, not fatty acids, as an energy source ${ }^{16}$ (Extended Data Fig. 
1b), and parietal cells require energy (NADPH) in the production of estrogen ( $3 \times \mathrm{NADPH}$ from testosterone in male, $6 \times \mathrm{NADPH}$ from progesterone in female, Fig. 1a). We therefore focused on the energy sources of parietal cells to obtain clues on how the production of gastric estrogen is regulated. Blood estrogen levels change in the menstrual cycle in females, so in this study we used male rats to exclude the effect of estrogen from the ovaries. Triglyceride (fatty acids) and glucose are major sources of energy for mammals. We examined the expression of the enzymes used in the generation of energy from fatty acids (acyl-CoA dehydrogenase medium chain [ACADM], an enzyme involved in b-oxidation) and glucose (glucokinase [GCK], a glycolytic enzyme) in the gastric mucosa (Figs. 1c, d). We identified the estrogen-producing cells and parietal cells using the antibodies against aromatase and $\mathrm{H}+\mathrm{K}+$ ATPase (ATP4B), respectively ${ }^{6}$. Aromatase-positive cells expressed high levels of ACADM, but little if any GCK. Conversely, aromatase-negative cells showed strong GCK but weak ACADM expressions (Fig. 1C). ATP4Bpositive parietal cells showed the same result as the aromatase-positive cells (Fig. 1d). We therefore conclude that parietal cells exclusively produce estrogen in the stomach and predominantly use fatty acids as an energy source.

Parietal cells secrete estrogen in an energy-dependent manner ${ }^{6,12}$ (Fig. 1a), and they predominantly use fatty acids as an energy source (Fig $1 \mathrm{c}$ and d). Moreover, estrogen from parietal cells directly enters the liver via the portal vein where lipogenesis occurs when blood triglyceride levels are $\operatorname{low}^{17}$, and it suppresses the increase of blood triglyceride levels by inhibiting the hepatic de novo lipogenesis ${ }^{9}$ and the feeding behavior ${ }^{8}$. We therefore hypothesized that parietal cells increase the secretion of estrogen as blood triglyceride levels rise, which suppresses the supply of lipid to maintain appropriate blood triglyceride levels.

To test this hypothesis, we designed a series of experiments. First, we investigated the relationship between the blood triglyceride and estrogen levels (Fig. 2). Triglyceride (olive oil, $2.5 \mathrm{ml} / \mathrm{kg}$ body weight) or control water was orally administered to rats and we measured their triglyceride and estrogen (E2) levels in the tail venous blood before $(0 \mathrm{~h})$ and at 1, 2, 3, 4, $5 \mathrm{~h}$ after the administration (Fig. 2a). Blood triglyceride levels (basal levels: $\sim 130 \mathrm{mg} / \mathrm{dL}$ ) increased, peaked at $2 \mathrm{~h}$ post-administration $(\sim 300 \mathrm{mg} / \mathrm{dL}$ ), and then declined to the basal levels $(5 \mathrm{~h})$ as reported previously ${ }^{18}$. Blood E2 levels (basal levels: $\sim 50$ $\mathrm{pg} / \mathrm{ml})$ also increased, peaked at $2 \mathrm{~h}$ after the administration $(\sim 80 \mathrm{pg} / \mathrm{ml})$, and then returned to the basal levels $(5 \mathrm{~h})$. Control rats did not show apparent changes in their blood triglyceride or in E2 levels. We then analyzed the relationship between the blood triglyceride and E2 levels at $2 \mathrm{~h}$ after the administration; there was a positive correlation between them (Fig. 2b). We further examined the effect of blood glucose on the blood estrogen levels. Glucose administration is known to decrease blood triglyceride levels via insulin-mediated inhibition of lipolysis in adipose tissues ${ }^{19,20}$, so we also monitored the blood triglyceride levels. Rats were orally administered glucose $(2 \mathrm{~g} / \mathrm{kg}$ body weight) or control water, and we measured their tail venous blood glucose, E2, and triglyceride levels before $(0 \mathrm{~h})$ and at $0.5,1,1.5,2,3,4,5 \mathrm{~h}$ after administration (Fig. 2c). Blood glucose levels (basal levels: $\sim 60 \mathrm{mg} / \mathrm{dL}$ ) increased, peaked at $1 \mathrm{~h}$ after the administration $(\sim 170 \mathrm{mg} / \mathrm{dL})$, and then declined toward the basal levels ${ }^{21}$. Blood E2 and triglyceride 
levels did not increase, however, they actually decreased when blood glucose levels were high. Blood estrogen levels are indicated to increase as blood triglyceride levels, but not glucose levels, rise.

Next, we investigated the effect of blood triglyceride levels on the production of estrogen in parietal cells. Parietal cells exclusively produce estrogen in the stomach and the elevated estrogen level in the portal vein compared with the artery is cancelled in GX rats $^{6}$. We therefore measured the E2 levels in the stomach tissues with the triglyceride levels in the tail venous blood at $2 \mathrm{~h}$ after the administration of olive oil or control water (Fig 3a). Rats administered olive oil showed elevated blood triglyceride ( $280 \mathrm{mg} / \mathrm{dL})$ and stomach E2 ( 3,500 pg/g tissue) levels compared with the controls (blood triglyceride: $\sim 110 \mathrm{mg} / \mathrm{dl}$, stomach E2: $1,900 \mathrm{pg} / \mathrm{g}$ tissue), and there was correlation between the blood triglyceride and stomach E2 levels. Parietal cells in the stomach were shown to produce estrogen in response to the blood triglyceride levels.

Finally, using GX rats, we investigated the involvement of the stomach in the regulation of blood estrogen levels by blood triglyceride levels (Fig. 3b, c). GX rats showed weaker increase of the blood triglyceride levels than normal rats when administered olive oil (data not shown), so we used $5 \mathrm{ml} / \mathrm{kg}$ body weight of olive oil in the studies using GX rats. Five days after total gastrectomy, GX rats were administered olive oil or control water. We measured triglyceride and E2 levels in the tail venous blood before ( $0 \mathrm{~h}$ ) and at 1, 2, 3, 4, $5 \mathrm{~h}$ after the administration (Fig. 3b). Blood triglyceride levels of $\mathrm{GX}$ rats (basal levels: $\sim 90 \mathrm{mg} / \mathrm{dL})$ increased, peaked at $2 \mathrm{~h}$ post-administration $(\sim 210 \mathrm{mg} / \mathrm{dL})$, and then declined to the basal levels ( $5 \mathrm{~h}$ ); but, blood E2 levels did not show apparent changes ( $60 \mathrm{pg} / \mathrm{ml})$. We also analyzed the relationship between the blood triglyceride and E2 levels at $2 \mathrm{~h}$ after the administration, but we found no correlation between them (Fig. 3c). The stomach is indicated to be the organ responsible for controlling the blood estrogen levels in response to the blood triglyceride levels.

In conclusion, we showed that parietal cells in the stomach predominantly produce estrogen among the tissues upstream of the portal vein. Production of estrogen requires energy, and parietal cells predominantly use triglyceride as the energy source. Blood estrogen levels increase as blood triglyceride levels rise, but not as glucose levels rise. Levels of estrogen in the stomach tissues increase as blood triglyceride levels rise, and in GX rats, increases of blood estrogen levels dependent on blood triglyceride are cancelled.

To maintain proper lipid conditions, hypothalamic NPY neurons collect the body lipid status to control hepatic lipogenesis and feeding behavior ${ }^{1}$. Ghrelin from the stomach, leptin from adipose tissues and vagus afferent nerves from the intestines and the liver report the levels of fasting, body fat and ingested lipids, respectively, and play a part in activation (ghrelin) or inhibition (leptin and vagus afferent nerves) of the NPY neurons ${ }^{2,4,5}$. Little is known, however, about the hormones or nerve fibers that inform the blood lipid levels. On the other hand, estrogen inhibits the hepatic de novo lipogenesis and the subsequent secretion of VLDL directly, ${ }^{9,223}$ or by suppressing the NPY neurons ${ }^{8,24,25}$. Little is known, however, about what information blood estrogen levels represent in terms of the lipid homeostasis. Among the organs upstream of the portal vein, the stomach and the pancreas do not take up nutrients 
and are suitable for sensing and directly informing the liver of the levels of "circulating" nutrients. Indeed, the pancreas secretes insulin or glucagon depending on the circulating blood glucose levels to control the blood glucose levels primarily by acting on the liver. We therefore collectively conclude that the stomach, a digestive organ, also acts as a blood triglyceride sensor organ, and parietal cells in the stomach secrete estrogen according to the blood triglyceride levels (Fig. 3d). When blood triglyceride levels rise, parietal cells increase the secretion of estrogen into the portal vein, which in turn lowers the blood triglyceride levels by inhibiting the hepatic de novo lipogenesis and feeding behavior. Conversely, when blood triglyceride levels lower, parietal cells decrease the secretion of estrogen to supply more lipid to the circulation.

This is the first demonstration of a mechanism by which gastric parietal cells sense the blood lipid levels and secrete estrogen to keep proper blood triglyceride levels by controlling the hepatic de novo lipogenesis and feeding behavior. Our model is consistent with previous reports on the daily rhythms of hepatic lipogenesis, blood triglyceride levels, and blood and gastric estrogen levels. Hepatic lipogenesis is regulated by daily feeding rhythm and rises when blood triglyceride levels are low ${ }^{17}$. Blood estrogen levels increase at night when rats eat actively, which is regulated by the secretion of gastric estrogen ${ }^{26}$.

Adipocytes dominantly use fatty acids as the energy source like parietal cells, and express high levels of ACADM (Extended Data Fig. 1c) and low levels of GCK (data not shown). Blood triglyceridedependent changes of blood estrogen levels are regulated by the stomach, but not by other tissues, such as adipose tissues. Adipocytes are filled with triglyceride, but parietal cells are not. Intracellular triglyceride levels are thus more susceptible to the extracellular (blood) triglyceride levels in parietal cells than in adipocytes. We therefore think parietal cells, not adipocytes, are suitable for the real-time monitoring of blood triglyceride levels.

As for gastric estrogen in females, parietal cells of female rats also secrete estrogen and increase the estrogen levels in the portal vein ${ }^{13}$; but estrogen and progesterone, a precursor for gastric estrogen in females, are mainly secreted from the ovaries. Moreover, the blood levels of estrogen and progesterone change dramatically with the menstrual cycle. The roles and regulation of gastric estrogen in females require further careful investigation.

\section{Materials And Methods}

\section{Animals}

Male Wistar rats were purchased from Kiwa Laboratory Animals Co., Ltd. (Wakayama, Japan). Animals were housed in an air-conditioned environment with $12 \mathrm{~h}$ light/dark cycle and allowed access to food (MF diet, Oriental Yeast Co. Ltd., Tokyo, Japan) and water ad libitum unless otherwise stated. All experiments were conducted according to the protocol approved by the Wakayama Medical University Animal Care and Use Committee.

\section{Immunoblotting}


Crude extracts of the stomach, duodenum, jejunum, ileum, colon and pancreas were obtained from adult male rats. Proteins (10 $\mu \mathrm{g}$ for aromatase, $1 \mu \mathrm{g}$ for b-actin) were separated by SDS-PAGE, transferred to a polyvinylidene fluoride membrane (Immobilon-P, Millipore) and detected with antibodies against aromatase (MCA2077S, BioRad) and b-actin (A5060, Sigma-Aldrich). Band intensities were quantified with LuminoGraph $\otimes$ (ATTO) using a standard enhanced chemiluminescence protocol. Processing of the images was performed with ImageJ 1.52 (National Institutes of Health).

\section{Fluorescence staining}

We performed immunostaining of rat tissue sections using a standard protocol ${ }^{27}$. Rats were anesthetized using isoflurane and fixed by perfusion with $4 \%$ paraformaldehyde in $0.1 \mathrm{M}$ phosphate buffer $(\mathrm{pH} 7.2)$. Tissues were then embedded in paraffin, sectioned at $5 \mu \mathrm{m}$ thickness using a microtome (model RX-860; Yamato Kohki Industrial), and subjected to heat-induced epitope retrieval treatment ( $\mathrm{pH}$ 6.0) according to the standard method. After blocking with Block One Histo (Nacalai Tesque), sections were incubated with the following primary antibodies: anti-aromatase, anti-ATP4B (MA3-923, Invitrogen), anti-ACADM (GTX100488, GeneTex), anti-GCK (HPA007093, Sigma-Aldrich), and anti-insulin (66198-1-lg, Proteintech). Sections were then incubated with Alexa Fluor-conjugated secondary antibodies from Thermo Fisher. After quenching the autofluorescence using TrueView from Vector Laboratory, samples were observed under an LSM-700 confocal laser-scanning microscope equipped with C-Apochromat 40/1.2W or PanApochromat 20/0.8 lens (Carl Zeiss). Images processing and analyses were performed with ImageJ 1.52 (National Institutes of Health).

\section{Gastrectomy}

Total gastrectomy (GX) was performed on 7-week-old male rats by anastomosing the duodenum and esophagus from end to end, as described elsewhere ${ }^{28}$. GX rats were used for the subsequent studies 5 days after the surgery.

\section{Oral administration of olive oil and glucose}

At 8 weeks of age, rats were deprived of food for $4 \mathrm{~h}$ before the experiment. Olive oil ("Yoshida", Yoshida Pharmaceutical Company, Tokyo, Japan) or control distilled water was orally administered, and blood samples from the tail vein were collected at 0 (before), and 1, 2, 3, 4 and $5 \mathrm{~h}$ after the administration ${ }^{18}$. GX rats showed weaker increase of blood triglyceride levels than normal rats when administered olive oil, so a smaller amount of olive oil was applied in the normal rats $(2.5 \mathrm{mg} / \mathrm{kg}$ body weight) compared with the $\mathrm{GX}$ rats ( $5 \mathrm{mg} / \mathrm{kg}$ body weight). In some studies, we sacrificed rats $2 \mathrm{~h}$ after the administration to obtain stomach tissues. Regarding oral administration of glucose, 8-week-old rats, deprived of food for $18 \mathrm{~h}$, were orally administered glucose $(2 \mathrm{~g} / \mathrm{kg}$ body weight, FUJIFILM Wako Pure Chemical Corp.) or control distilled water ${ }^{21}$. Blood samples from tail veins were collected at 0 (before) and 0.5, 1, 1.5, 2, 3, 4 and $5 \mathrm{~h}$ after the administration. We excluded the rats whose tail venous triglyceride levels prior to the administration were higher than $200 \mathrm{mg} / \mathrm{dL}$. 


\section{Measurement of triglyceride, estrogen and glucose concentrations}

The plasma triglyceride concentrations were measured using a LabAssay Triglyceride (FUJIFILM) according to the manufacturer's instructions. The total estrogen (E2) concentrations in the plasma fractions of blood samples and the stomach tissues ${ }^{29}$ were determined using an Estradiol ELISA kit (Cayman Chemical Company, Ann Arbor, MI, USA) according to the manufacturer's instruction. Blood glucose levels were measured using FreeStyle Libre (Abbott Japan) according to the manufacturer's instructions.

\section{Statistical analysis}

Statistical analyses were performed using JMP Pro ver. 14 (SAS Institute Japan). $P$ values $<0.05$ were considered to be significant.

\section{Method References}

27. Kanai, Y., Wang, D. \& Hirokawa, N. KIF13B enhances the endocytosis of LRP1 by recruiting LRP1 to caveolae. 204, 395-408 (2014).

28. Ueyama, T. et al. Is Gastrectomy-Induced High Turnover of Bone with Hyperosteoidosis and Increase of Mineralization a Typical Osteomalacia? PLOS ONE 8, e65685-13 (2013).

29. Bligh, E. G. \& Dyer, W. J. A rapid method of total extraction and purification of lipids. Can J Biochem Physio/ 37, 911-917 (1959).

\section{Declarations}

Data availability There is no accession code, unique identifier, or special data set used in this study.

Acknowledgements We thank Yuki Yamazaki, Shota Nasu, Yamato Tsunoda and Keigo Matsui from the Kanai Laboratory for technical assistance. We acknowledge proofreading and editing by Benjamin Phillis at Wakayama Medical University. This study was supported by Medical Research Support from Wakayama Medical University (to Y.K.). This paper is dedicated to the late professor Takashi Ueyama (Wakayama Medical University, Wakayama, Japan).

Author contributions Y.K. conceived the project, invented the hypothesis, designed the experiments, wrote the manuscript, and made the figures including the drawing of the model diagram. Y.Y. and N.Y. performed immunoblot studies. Y.K. and T.I. performed immunofluorescence studies. T.I. generated GX rats. T.I. and Y.Y. performed olive oil and glucose adiminstration experiments. T.I. measeured blood TG and blood and stomach E2 levels. T.I. and Y.Y. measeured blood glucose levels. Y.Y. performed statistical analysis. T.I, Y.Y. and N.Y. contributed equally.

Competing interests The authors declare no competing interests. 


\section{References}

1. Keesey, R. E. \& Powley, T. L. Body energy homeostasis. Appetite 51, 442-445 (2008).

2. Sato, T. et al. Structure, regulation and function of ghrelin. J Biochem 151, 119-128 (2012).

3. Matschinsky, F. M. \& Wilson, D. F. The Central Role of Glucokinase in Glucose Homeostasis: A Perspective 50 Years After Demonstrating the Presence of the Enzyme in Islets of Langerhans. Front. Physiol. 10, 389-15 (2019).

4. Ahima, R. S. \& Flier, J. S. Leptin. Annu. Rev. Physiol. 62, 413-437 (2000).

5. Waise, T. M. Z., Dranse, H. J. \& Lam, T. K. T. The metabolic role of vagal afferent innervation. Nat. Rev. Gastroenterol. Hepatol. 1-12 (2018). doi:10.1038/s41575-018-0062-1

6. Ueyama, T. et al. Gastric parietal cells: potent endocrine role in secreting estrogen as a possible regulator of gastro-hepatic axis. Endocrinology. 143, 3162-3170 (2002).

7. Patel, S., Homaei, A., Raju, A. B. \& Meher, B. R. Estrogen: The necessary evil for human health, and ways to tame it. Biomed. Pharmacother. 102, 403-411 (2018).

8. Dhillon, S. S. \& Belsham, D. D. Estrogen inhibits NPY secretion through membrane-associated estrogen receptor (ER)-a; in clonal, immortalized hypothalamic neurons. Int. J. Obes. 1-10 (2019). doi:10.1038/ijo.2010.124

9. Qiu, S. et al. Hepatic estrogen receptor a is critical for regulation of gluconeogenesis and lipid metabolism in males. Sci. Rep. 1-12 (2017). doi:10.1038/s41598-017-01937-4

10. Simpson, E. R. Sources of estrogen and their importance. J. Steroid. Biochem. Mol. Biol. 86, 225-230 (2003).

11. Bracht, J. R. et al. The role of estrogens in the adipose tissue milieu. Ann. N. Y. Acad. Sci. 1461, 127143 (2019).

12. Ueyama, T., Shirasawa, N., Ito, T. \& Tsuruo, Y. Estrogen-producing steroidogenic pathways in parietal cells of the rat gastric mucosa. Life Sci. 74, 2327-2337 (2004).

13. Kobayashi, H., Yoshida, S., Sun, Y.-J., Shirasawa, N. \& Naito, A. Changes of gastric aromatase and portal venous $17 \mathrm{~b}$-estradiol during the postnatal development and estrus cycle in female rats. Endocrine 46, 605-614 (2013).

14. Müller, T. D., Finan, B., Clemmensen, C., DiMarchi, R. D. \& Tschöp, M. H. The New Biology and Pharmacology of Glucagon. Physiol. Rev. 97, 721-766 (2017).

15. Tokarz, V. L., MacDonald, P. E. \& Klip, A. The cell biology of systemic insulin function. J. Cell Biol. 217, 2273-2289 (2018).

16. Matschinsky, F. M. Glucokinase as glucose sensor and metabolic signal generator in pancreatic betacells and hepatocytes. Diabetes 39, 647-652 (1990).

17. Su, Y., Foppen, E., Machado, F. S. M., Fliers, E. \& Kalsbeek, A. The role of the daily feeding rhythm in the regulation of the day/night rhythm in triglyceride secretion in rats. Chronobiol. Int. 35, 885-895 (2018). 
18. García-Martín, R. et al. Adipocyte-Specific Hypoxia-Inducible Factor 2a Deficiency Exacerbates Obesity-Induced Brown Adipose Tissue Dysfunction and Metabolic Dysregulation. Mol. Cell. Biol. 36, 376-393 (2016).

19. Spégel, P. et al. Metabolomic analysis of a human oral glucose tolerance test reveals fatty acids as reliable indicators of regulated metabolism. Metabolomics 6, 56-66 (2009).

20. Ruud, J., Steculorum, S. M. \& ning, J. C. B. U. Neuronal control of peripheral insulin sensitivity and glucose metabolism. Nat. Commun. 8, 1-12 (2017).

21. Miyamoto, J. et al. Gut microbiota confers host resistance to obesity by metabolizing dietary polyunsaturated fatty acids. Nat. Commun.1-15 (2019). doi:10.1038/s41467-019-11978-0

22. Chow, J. D. Y., Jones, M. E. E., Prelle, K., Simpson, E. R. \& Boon, W. C. A selective estrogen receptor a agonist ameliorates hepatic steatosis in the male aromatase knockout mouse. J. Endocrinol. 210, 323-334 (2011).

23. Lee, Kim \& Jung. Potential Therapeutic Application of Estrogen in Gender Disparity of Nonalcoholic Fatty Liver Disease/Nonalcoholic Steatohepatitis. Cells 8, 1259-20 (2019).

24. Stafford, J. M. et al. Central Nervous System Neuropeptide Y Signaling Modulates VLDL Triglyceride Secretion. Diabetes 57, 1482-1490 (2008).

25. Geerling, J. J. et al. Sympathetic nervous system control of triglyceride metabolism: novel concepts derived from recent studies. J. Lipid Res. 55, 180-189 (2014).

26. Kobayashi, H., Yoshida, S., Sun, Y.-J., Shirasawa, N. \& Naito, A. Gastric $17 \beta$-estradiol in portal vein and liver Esr1 make a circadian rhythm in systemic circulation in male rats. Endocrine 53, 565-573 (2016).

\section{Figures}


a

progesterone (female)

17a-hydroxylase

$17 \alpha \mathrm{OH}$-progesterone

$\downarrow \begin{gathered}17 \alpha \text {-hydroxylase } \\ \text { (NADPH) }\end{gathered}$

androstenedione

$\downarrow \begin{gathered}17 \beta-H S D \text { type III } \\ \text { (NADPH) }\end{gathered}$
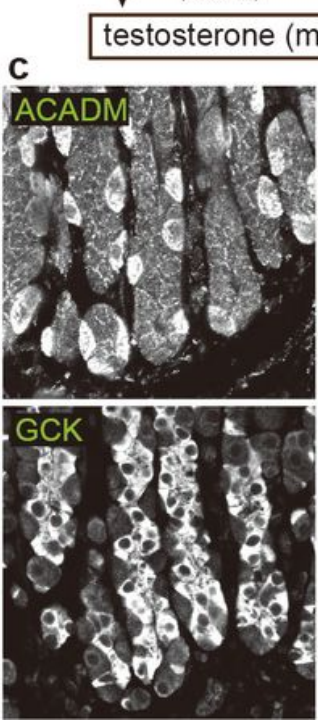

b

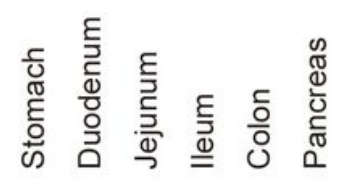

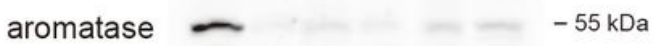

estrone (E1)

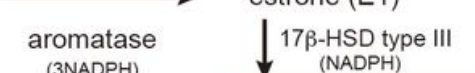

(3NADPH)

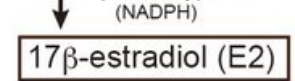

$17 \beta$-estradiol (E2)

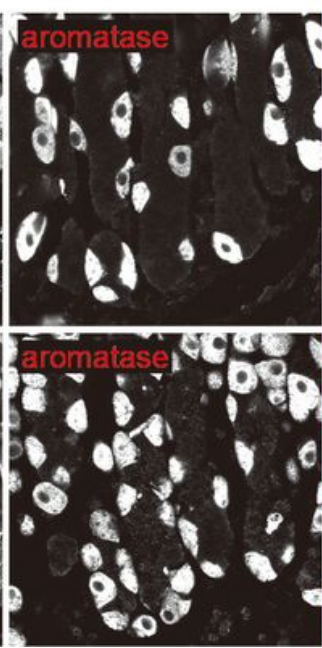

$-55 \mathrm{kDa}$
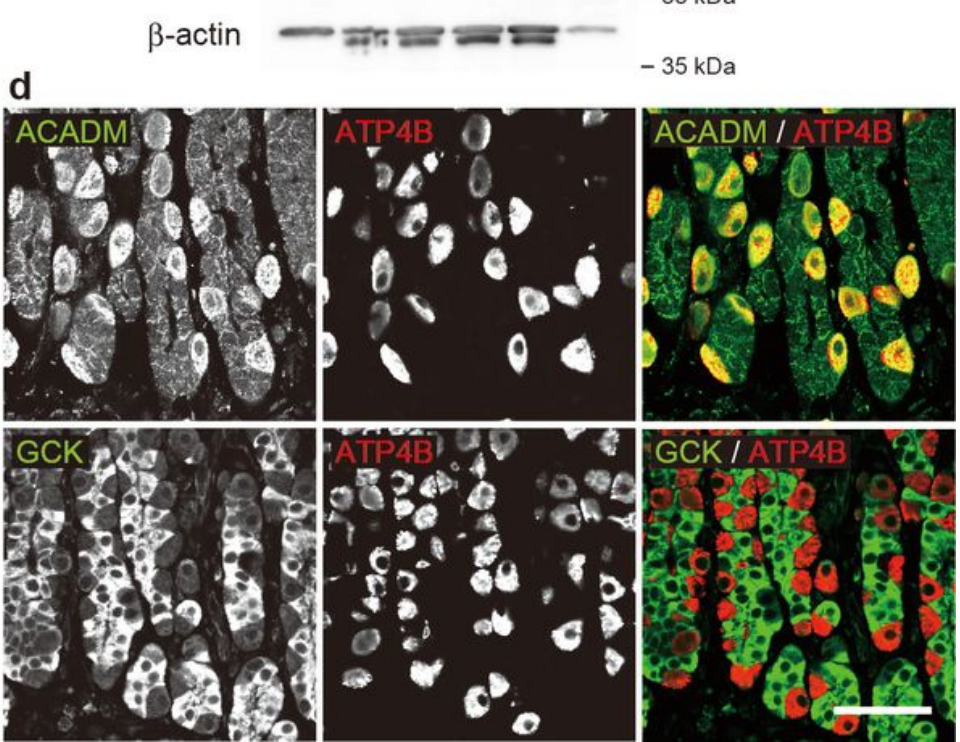

\section{Figure 1}

Estrogen producing gastric parietal cells use fatty acid as energy source a, Scheme of testosterone (progesterone)-17ß-estradiol (E2) conversion pathway in rat gastric parietal cells. b, Immunoblotting of crude extracts from the stomach, duodenum, jejunum, ileum, colon and pancreas of adult male rats using antibodies against aromatase and control $\beta$-actin. $c$, Rat gastric mucosa was double stained for ACADM (acyl-CoA dehydrogenase medium chain, an enzyme involved in $\beta$-oxidation) or GCK (glucokinase, a glycolytic enzyme) (green) with aromatase (red). d, Rat gastric mucosa was double stained for ACADM or GCK (green) with ATP4B (H+/K+ ATPase, a gastric parietal cell marker; red). Bars: $50 \mu \mathrm{m}$. 
a

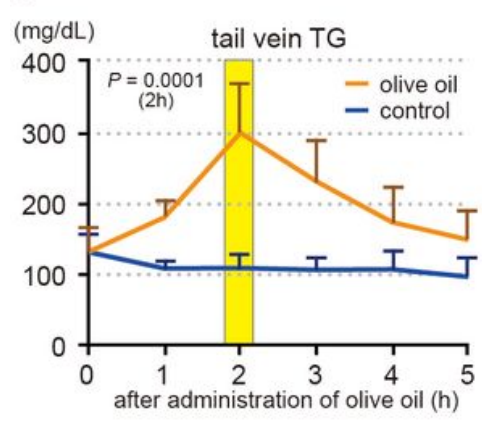

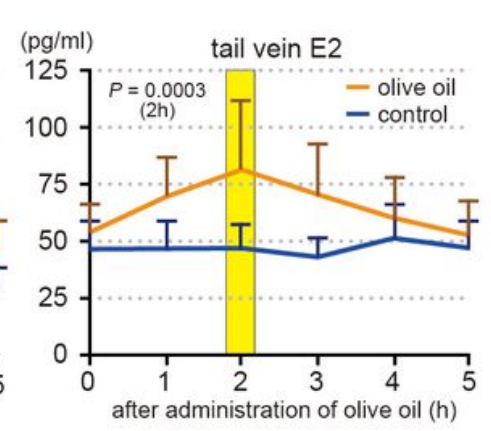

C
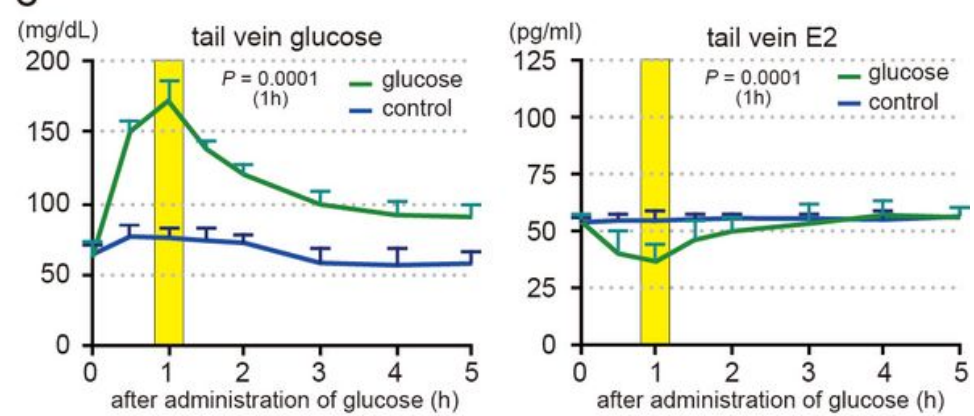

$\mathrm{b}$
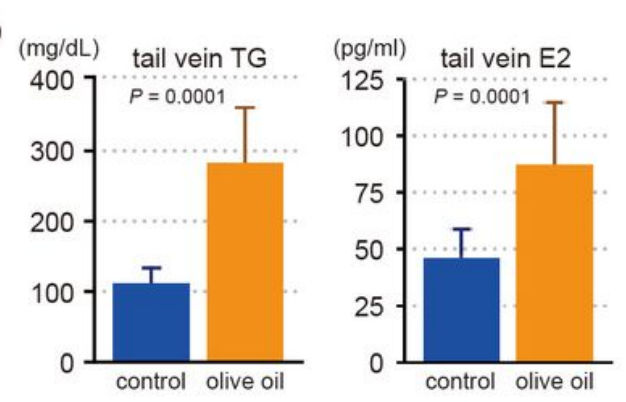

$2 \mathrm{~h}$ after administration of olive oil
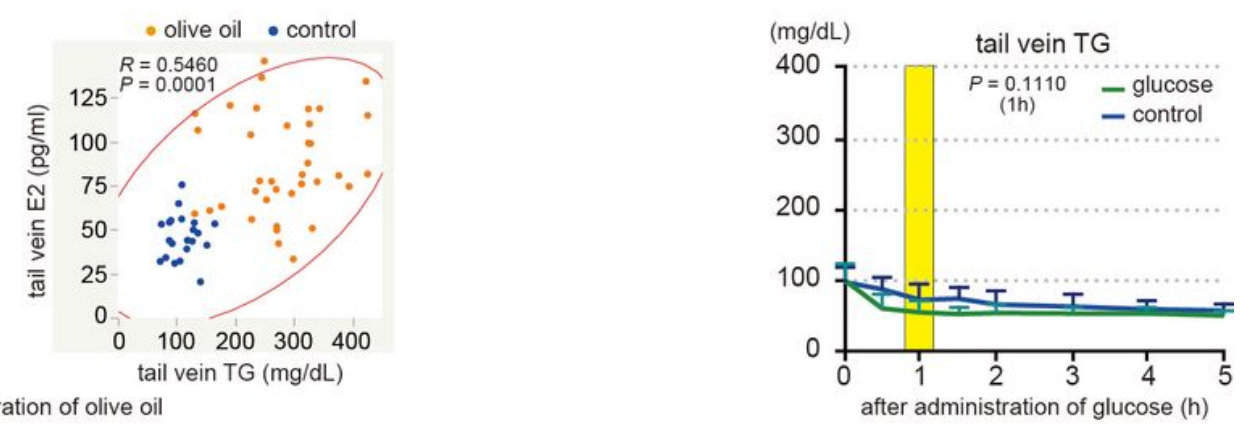

Figure 2

Blood estrogen levels increase as blood triglyceride levels, but not glucose levels, rise a, Rats were orally administered olive oil $(n=13)$ or control water (control, $n=8)$. TG and E2 levels in the tail venous blood measured before ( 0 ) and at 1, 2, 3, 4, $5 \mathrm{~h}$ after the administration. b, TG and E2 levels in the tail venous blood measured at $2 \mathrm{~h}$ after the administration of olive oil $(n=37)$ or control water $(n=21)$ (left), and the correlation diagram between the blood TG and E2 levels ( $n=58$, right). c, Rats were orally administered glucose $(n=10)$ or control water $(n=9)$. Glucose, E2 and TG in the tail venous blood measured before $(0)$ and at $0.5,1,1.5,2,3,4,5 \mathrm{~h}$ after the administration. $\mathrm{n}$, number of rats. Data are mean $\pm \mathrm{s}$.d. $\mathrm{P}$ values determined by two-sided Student's t-test at $2 \mathrm{~h}(\mathrm{a}, \mathrm{b}$, left) or $1 \mathrm{~h} \mathrm{(c)} \mathrm{after} \mathrm{the} \mathrm{administration.} \mathrm{b}$, right, $\mathrm{R}$ and $P$ values determined by Pearson's product moment correlation with $95 \%$ density ellipse. 

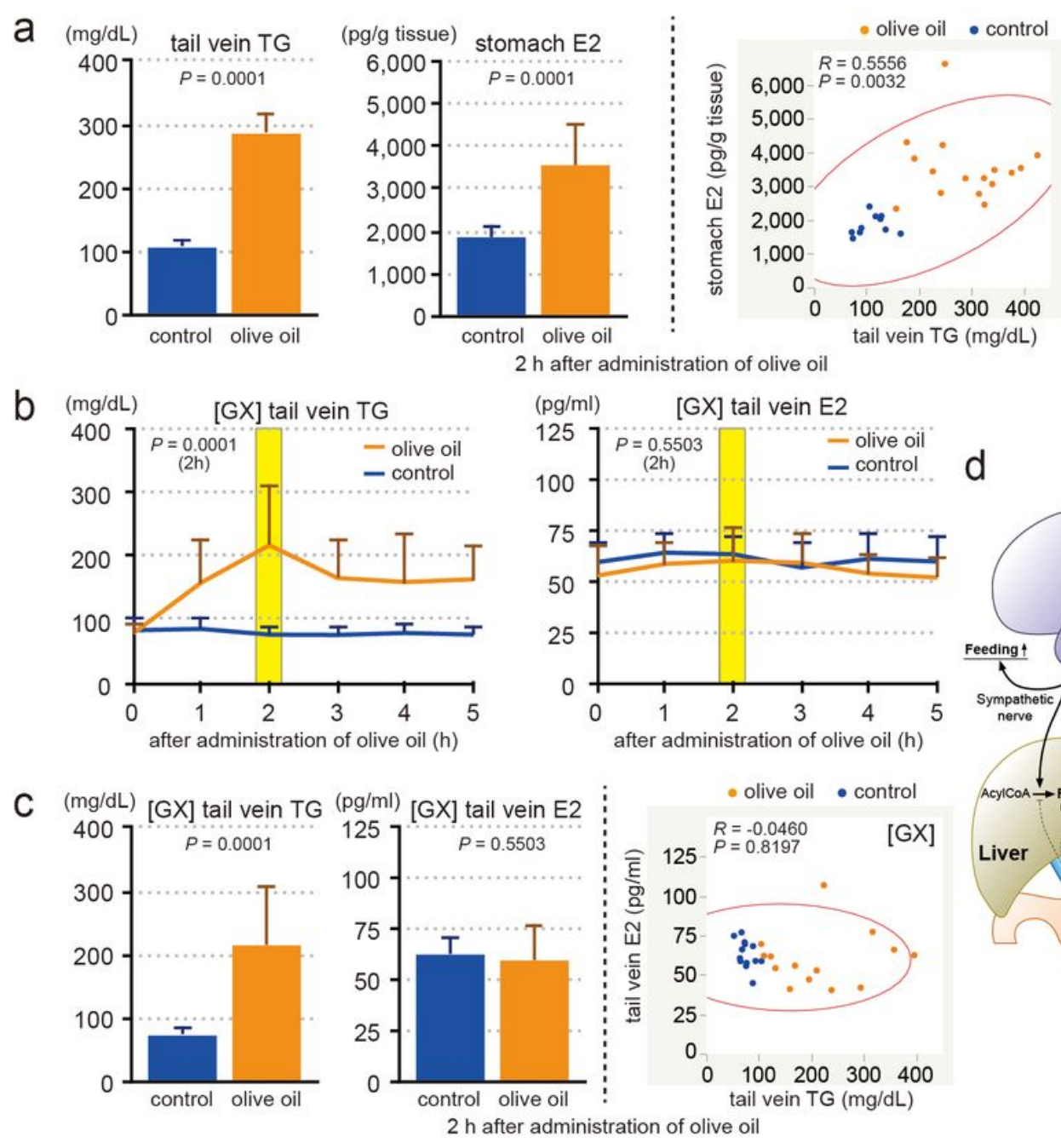

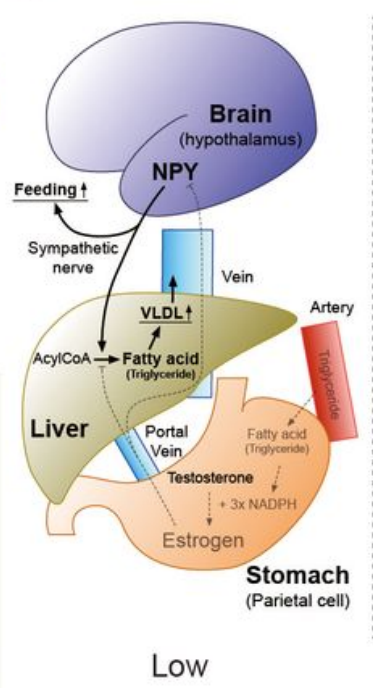

Low

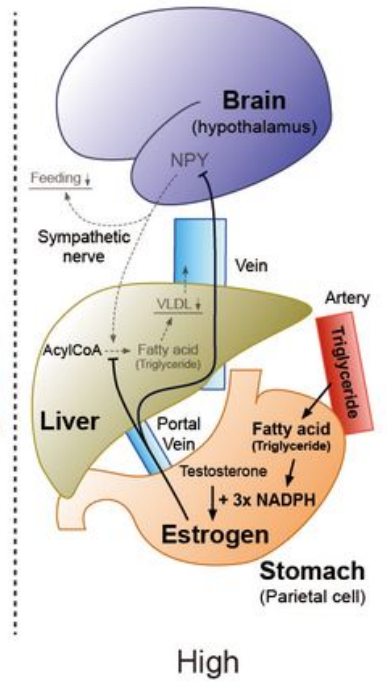

Blood triglyceride levels

\section{Figure 3}

Stomach secretes estrogen in response to the blood triglyceride levels a, Rats were orally administered olive oil $(n=16)$ or control water $(n=10)$. TG levels in the tail venous blood and E2 levels in the stomach tissues measured at $2 \mathrm{~h}$ after the administration (left), and the correlation diagram between the blood TG and stomach E2 levels ( $n=26$, right). b, GX rats were orally administered olive oil $(n=14)$ or control water $(n=13)$. TG and E2 levels in the tail venous blood measured before ( 0 ) and at 1, 2, 3, 4, $5 \mathrm{~h}$ after the administration. $\mathrm{c}, \mathrm{TG}$ and $\mathrm{E} 2$ levels in the tail venous blood of $\mathrm{GX}$ rats measured at $2 \mathrm{~h}$ after the administration of olive oil $(n=14)$ or control water $(n=13)$ (left), and the correlation diagram between the blood TG and E2 levels ( $n=27$, right). $d$, Proposed model for the role of gastric estrogen in the control of blood triglyceride levels. When blood triglyceride levels rise, parietal cells in stomach increase the secretion of estrogen (this study), which suppresses the hepatic lipogenesis and the feeding behavior directly or by inhibiting the hypothalamic NPY neurons8,9. n, number of rats. Data are mean \pm s.d. a, left, b, c, left, $\mathrm{P}$ values determined by two-sided Student's t-test at $2 \mathrm{~h}$ after the administration. a, right, $\mathrm{c}$, right, $\mathrm{R}$ and $\mathrm{P}$ values determined by Pearson's product moment correlation with $95 \%$ density ellipse.

\section{Supplementary Files}


This is a list of supplementary files associated with this preprint. Click to download.

- ExtendedDatafig1.png 